\title{
5 STUDY OF TECHNICAL SYSTEMS OF MATERIALS 5 COMPACTION PROCESS
}

\begin{abstract}
I. Nazarenko, O. Dedov, A. Bondarenko, A. Zapryvoda, M. Kyzminec, M. Nazarenko, M. Ruchynskyi, A. Svidersky, V. Slipetskyi

ABSTRACT
\end{abstract}

The working process of concrete mixture compaction is investigated. The model of the processing medium is determined, which is characterized by three stages. At the first stage of compaction it is a free-flowing medium, at the second and third stages of compaction it is an elastic-viscoplastic medium. This result lies in the definition of the physical and mathematical model, the equations of motion. The vibration system "machine-medium» at any moment of movement consists of two components - one is capable of accumulating energy that passes from one form to another (reactance), and the second is energy dissipation (active resistance). The influence of active and reactive forces on the general movement of the system under study has been investigated and determined. By solving the equations, analytical dependencies were obtained, which established the laws of motion of the "Vibration machine - sealing medium» system. Practical implementation for technical systems for the formation of concrete products and soil compaction processes under the main pipeline has been carried out. An algorithm and method for calculating the main parameters of vibration systems for compaction of working media have been developed.

\section{KEYWORDS}

Vibration system, medium, compaction process, concrete mix, soil, physical, mathematical model, reactance, active resistance, equation, analytical dependences, parameters, experimental units, amplitude, vibration frequency.

\subsection{DETERMINATION OF PHYSICAL MODELS OF THE PROCESS OF VIBRATION COMPACTION OF MATERIALS}

The vibration effect on the concrete mix is of great practical importance and forms the basis of all modern technology for compaction of consistencies [1-3]. The essence of the vibration action lies in the fact that during vibrations, the concrete mixture acquires the properties of fluidity due to the violation of bonds between the particles. The compaction process is characterized by the following movement of concrete mixture particles: individual, general (general) vibration of concrete mixture particles as a continuous system. The uniform movement of particles from one equilibrium position to another occurs predominantly in the surface layer. 
Based on a general view of the compaction process, it can be noted that the concrete mix at different stages obeys different laws. At first, it can be represented as a free-flowing medium, where the vibration movement of its particles occurs, that is, the forces of dry friction mainly affect. With subsequent compaction, the mixture becomes more mobile and behaves like an elastic viscoplastic body. Along with the properties of a viscous liquid, the mixture is clearly elastic. Thus, the physical model of the mixture should take into account three stages of the compaction process (Fig. 5.1).

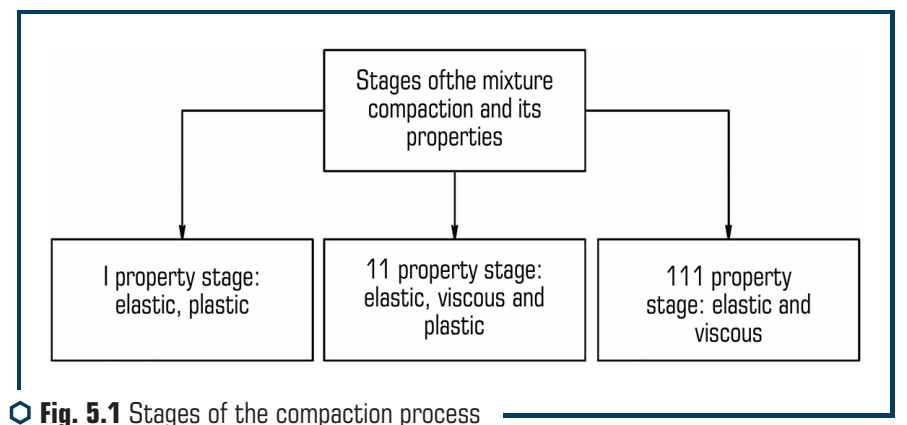

The elastic properties according to Hooke's law (Table 5.1) are presented in the form of a linear (linear model) or nonlinear (nonlinear model) layer of a concrete mixture.

Viscous properties according to Newton's law (Table 5.1) are modeled by a damper consisting of a piston moving in a cylinder with a liquid. Other connections of elements with each other are possible, which is due to the need to take into account certain properties and composition of the components of a real mixture. The equations describing the propagation of a wave process in a mixture can be represented as follows:

$$
\frac{\partial^{2} u}{\partial t^{2}}=c^{2} \frac{\partial^{2} u}{\partial z^{2}} ; \frac{\partial^{2} u}{\partial t^{2}}=c^{2} \frac{\partial^{2} u}{\partial z^{2}}+\frac{\eta}{\rho} \frac{\partial^{3} u}{\partial^{2} z \partial t} ; \frac{\partial^{2} u}{\partial t^{2}}=c^{2}(1+i \gamma) \frac{\partial^{2} u}{\partial z^{2}} .
$$

The first equation (5.1) describes the displacement of the layer of the mixture $u(z, t)$ as an elastic body with density at a speed $c$. The second equation (5.1) describes the motion of the mixture as an elastic-viscous body with density with a frequency-dependent dissipation of energy taken into account in the equation by the coefficient $\eta$. The third equation (5.1) describes the motion of the mixture as an elastic - viscous body with density $\rho$ with a frequency-independent dissipation of energy taken into account in the equation by the coefficient $\gamma$. At different stages of the concrete compaction process (Fig. 5.1), all equations (5.1) can be applied. The first equation (5.1) is not applicable in the case of a possible resonance, since the absence of a dissipative component in the resonance zone gives a solution for the amplitude of oscillations $A \rightarrow \infty$. Therefore, the second and third equations (5.1) are left to choose from. At the present stage of development of vibration 
technology for compaction of building mixtures, the third equation (5.1) is more applied due to the fact that for a specific vibration frequency, the stress state is determined by the vibration amplitude. Therefore, in further analytical studies, the third equation (5.1) is used.

Table 5.1 The main types of physical models

\begin{tabular}{|c|c|c|c|}
\hline \multirow{2}{*}{$\begin{array}{l}\text { Model } \\
\text { properties }\end{array}$} & \multicolumn{2}{|c|}{ Physical model } & \multirow{2}{*}{ Model name } \\
\hline & scheme & Mathematical notation & \\
\hline Elastic & & $\sigma=\varepsilon \mathrm{E} ; \tau=x G$ & Hooke \\
\hline Plastic & & $\tau=\tau_{p l}$ & Saint-Venant \\
\hline Elastic-plastic & & $\tau=\tau_{p l} ; \quad \tau=x G$ & Prandtl \\
\hline Viscous & & $\tau=\eta \frac{d V}{d z}$ & Newton \\
\hline $\begin{array}{l}\text { Elastoviscous with } \\
\text { stress relaxation }\end{array}$ & & $\tau=\eta \frac{d V}{d z} ; \tau=x G$ & Maxwell \\
\hline $\begin{array}{l}\text { Elastoviscous with } \\
\text { delayed deforma- } \\
\text { tions }\end{array}$ & & $\tau=x G+\eta \frac{d V}{d z}$ & Voigt \\
\hline $\begin{array}{l}\text { Elastoviscous with } \\
\text { creep phenomenon }\end{array}$ & & $\tau=x G ; \quad \tau=x_{1} G_{1}+\eta \frac{d V}{d z}$ & Kelvin \\
\hline Elasto-visco-plastic & & $\tau=x G ; \quad \tau=x_{1} G_{1}+\tau_{p l}$ & Bingham \\
\hline $\begin{array}{l}\text { Elasto-visco-plastic } \\
\text { with relaxation }\end{array}$ & & $\tau=x G ; \quad \tau=x_{1} G_{1}+\tau_{p l} ; \quad \tau=\tau_{p l}+\eta \frac{d V}{d z}$ & Shvedov \\
\hline $\begin{array}{l}\text { Elasto-visco-plastic } \\
\text { in the process of } \\
\text { shear deformation }\end{array}$ & & $\tau=x G ; \quad \tau=x_{1} G_{1}+\eta \frac{d V}{d z} ; \tau=\tau_{p l}+\eta_{1} \frac{d V}{d z}$ & $\begin{array}{l}\text { Generalized } \\
\text { Kelvin and } \\
\text { Shvedov }\end{array}$ \\
\hline
\end{tabular}

When vibrations are transmitted from the working body to the mixture (Fig. 5.2), elastic waves propagate in the latter, which carry the energy that goes to the compaction of the mixture layers. 


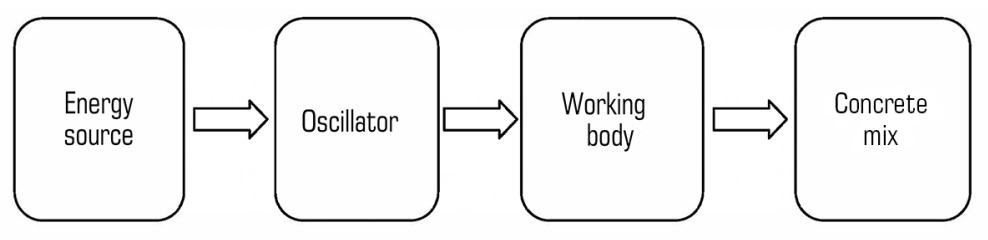

Fig. 5.2 «Vibration unit - concrete mix» block diagram

Based on the above reasoning, the "machine - medium» vibration system by different signs of their behavior will be represented from the point of view of modeling as subsystems with discrete (machine) and distributed (medium) parameters.

The next premise is based on the fact that the «machine - medium» vibration system at any moment of movement consists of two components - one is capable of accumulating energy that passes from one form to another (reactance), and the second is energy dissipation (active resistance) [3].

Thus, the task of accounting for the medium to be processed is reduced to determining the active and reactive forces in the contact zone and introducing them into the equation of motion of the vibration unit.

For this purpose, the «vibration unit - concrete mix» design diagram according to the method [2] is shown in Fig. $\mathbf{5 . 3}$.

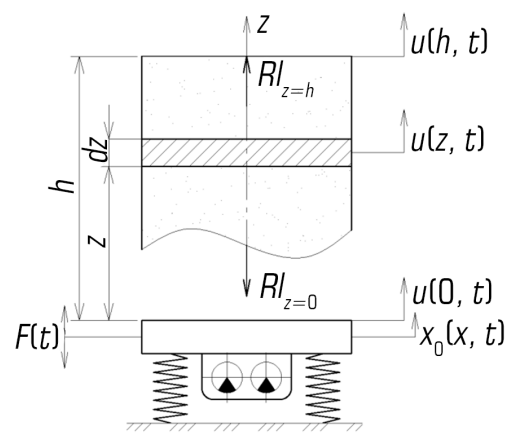

O Fig. 5.3 Design diagram of the "vibration plant - concrete mix» system:

$x$ - displacement; $F(t)$ - external forced force; $u(z, t)$ - longitudinal displacement of the current section of the concrete mixture column; $R_{z=0}$ - reaction of the concrete mixture in the section, provided that $z=0 ; R_{z=h}$ - reaction of the concrete mixture in the cross section of the column, provided that $z=h$ 
The wave equation of displacement of the current section of the column of concrete mixture is taken in the form (5.1):

$$
\frac{\partial^{2} u}{\partial t^{2}}=c^{2}(1+i \gamma) \frac{\partial^{2} u}{\partial z^{2}}
$$

Here $\partial^{2} u / \partial t^{2}$ - acceleration of the concrete mixture layer; $\partial^{2} u / \partial z^{2}$ - the second derivative of the displacement of the concrete mixture layer $u$ along the coordinate of the mixture column $z$; $c$ - speed of the mixture layer; $\gamma$ - coefficient of resistance of the concrete mixture, which characterizes the quantitative value of energy dissipation; $i$ - imaginary unit showing the rotation between the elastic and dissipative components by the angle $\varpi / 2$.

The solutions of the wave equation (5.1) will be used in the form:

$$
u(z, t)=\left(u_{1} e^{k z}+u_{2} e^{-k z}\right) e^{i \omega t} .
$$

The displacement $u$ is determined by the product of two functions, one of which depends on the arguments $z(z)$ and $T(t)$ :

$$
\begin{aligned}
& z(z)=u_{1} e^{k z}-u_{2} e^{-k z}, \\
& T(t)=e^{i \omega t} .
\end{aligned}
$$

In solution (5.2) $u_{1}$ and $u_{2}$ are constants determined by the boundary conditions, $k$ - complex wavenumber: $k=(\alpha+i \beta)$, where $\alpha, \beta$ - coefficients [1-3]:

$$
\alpha=\mu \frac{\omega}{c_{2}} ; \beta=v \frac{\omega}{c_{2}},
$$

where $\mu=\sqrt{\frac{\sqrt{1+\gamma^{2}}-1}{2\left(1+\gamma^{2}\right)}} ; v=\sqrt{\frac{\sqrt{1+\gamma^{2}}+1}{2\left(1+\gamma^{2}\right)}}$.

The coefficient $\alpha$ determines the attenuation of the wave propagated in the layer of the medium, and the coefficient $\beta$ determines the attenuation of the same wavelength. Indeed, if there is no resistance $(\gamma=0)$, then $\alpha=0, \beta=\omega / c_{0}$, that is, the wave under this condition propagates without extinction, which corresponds to the first equation (5.1).

The main task is to provide an analytical description of the dynamics of the "vibration unit concrete mix» hybrid system.

The design diagram of the vibration system (Fig. 5.4) is described by the equation obtained on the basis of the use of the reaction method [4-6]:

$$
\left(m+m_{c} a\right) \ddot{x}+\left(b+m_{c} \omega d\right) \dot{x}+c_{0} x=F_{0} \cdot \sin \omega t .
$$


The solution to this equation gives an expression $x=x_{0} \sin (\omega t+\gamma)$ for determining the vibration amplitude of a vibration machine, taking into account the entire complex of resistance forces:

$$
x_{0}=\frac{F_{0}}{\sqrt{\left[\left(c-m w^{2}\right)+m_{c} \omega^{2} a\right]^{2}+\left(b \omega+m_{c} w^{2} d\right)^{2}}},
$$

where $a$ - wave coefficient of the reactance of the concrete mixture; $d$-wave coefficient of the active resistance of the concrete mixture;

$$
a=\frac{\alpha \operatorname{sh} 2 \alpha h+\beta \sin 2 \beta h}{h\left(\alpha^{2}+\beta^{2}\right)[\operatorname{ch} 2 \alpha h+\cos 2 \beta h]} ; d=\frac{\alpha \sin 2 \beta h-\beta \operatorname{sh} 2 \alpha h}{h\left(\alpha^{2}+\beta^{2}\right)[\operatorname{ch} 2 \alpha h+\cos 2 \beta h]} .
$$

Because of the task at hand, it is important to determine not only the influence of the nominal resistance forces on the amplitude value of displacement in the contact zone, but also to find an expression for determining the nature and magnitude of the distribution of the amplitudes of displacement over the layers of the mixture $z=z(h)$.

Using the solution of the wave equation, let's find an expression for finding the displacement of any layer of the mixture:

$$
x\left(z_{1} t\right)=\frac{x_{0} \operatorname{ch}(h-z)\left(\alpha_{1}+i \beta_{1}\right)}{\operatorname{ch}\left[h\left(\alpha_{1}+i \beta_{1}\right)\right]} e^{i(w t+\varphi)} .
$$

The modulus of expression (5.8) gives a formula for the amplitude of the displacement of the mixture layer:

$$
x(z, t)=\frac{x_{0} \operatorname{ch}(h-z)\left(\alpha_{1}+i \beta_{1}\right) e^{i(w t+\varphi)}}{\operatorname{ch}\left[h\left(\alpha_{1}+i \beta_{1}\right)\right]} .
$$

When to enter an additional element, for example when loading, the limit conditions are changed. Now the stress on the upper contacting surface will not be equal to zero. It is obvious that the surcharge, like the working body, in its motion will also perceive the influence of the active and reactive resistances of the medium. By analogy, the force of resistance to surcharge fluctuations can be expressed as:

$$
\begin{aligned}
& R(h, t)=E S(1+i \gamma)\left(\frac{\partial u}{\partial z}\right)_{z=h}= \\
& =\rho c_{b}^{2} S\left(\frac{k^{2}}{\delta^{2}}\right) \delta\left[u_{1} e^{z \delta}-u_{2} e^{-z \delta}\right]_{z=h} e^{i \omega t}=\frac{m_{c} w^{2}}{h} \cdot \frac{e^{i \omega t}}{\delta}\left(u_{1} e^{h \delta}-u_{2} e^{-h \delta}\right) .
\end{aligned}
$$

The notation is accepted here $k=w / c ; m_{A}=\rho S h ; \delta=\alpha+i \beta$. 


\subsection{INVESTIGATION OF THE PARAMETERS OF VIBRATION MACHINES FOR THE COMPACTION OF CONCRETE MIX}

To study the parameters of vibration machines for the compaction of a concrete mixture according to the obtained formulas, calculations were made according to the following variable parameters: the height of the mixture column $h=0.1 \ldots 1.5 \mathrm{~m}$; the frequency of the oscillations $w=100 . .300 \mathrm{1} / \mathrm{c}$; the speed of propagation of vibrations in the concrete mixture $C_{c}=30 \ldots 50 \mathrm{~m} / \mathrm{s}$; the loss factor $\gamma=0.05 \ldots 1.0$; the ratio of the masses of the concrete mixture $m_{c}$ to the mass of the vibration machine $m: m_{c} / m=0.4 \ldots 1.0$; static pressure. Fig. $\mathbf{5 . 4}$ shows the results calculations.

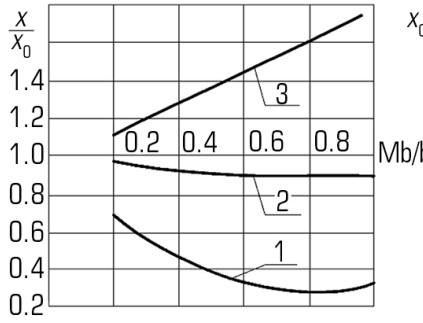

$a$

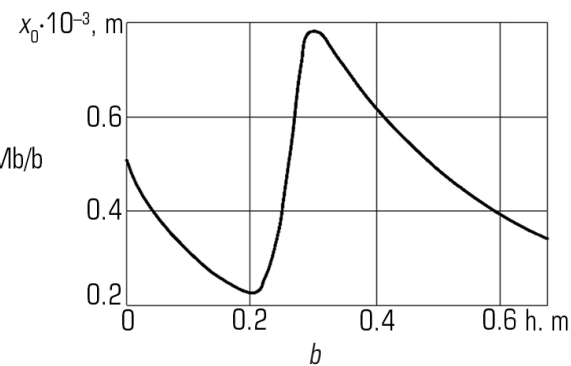

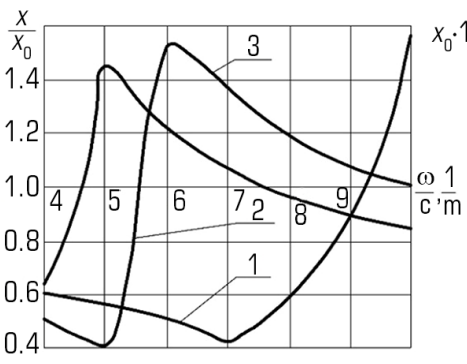

C

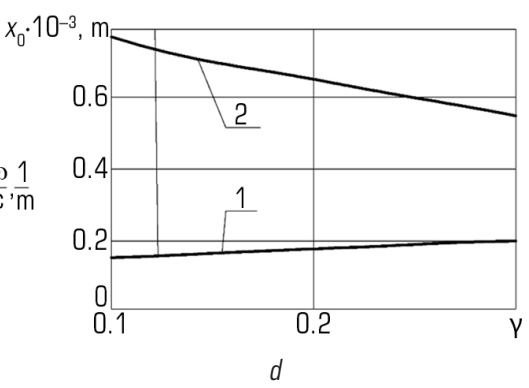

$d$

O Fig. 5.4 The results of calculations of changes in the parameters of vibration machines for the processes of compaction of a concrete mixture: $a-$ the influence of the ratio of the masses of the medium and the machine on the amplitude of oscillations; $b$ - the dependence of the amplitude of the oscillations on the height of the mixture column; $c$ - the influence of the wave number on the amplitude of the oscillations; $d$ - the influence of the loss factor on the vibration amplitude of the working body

The effect of the mass ratio of the medium and the machine on the vibration amplitude depends on the specific height of the mixture column. So, in zones far from resonance, these are the heights of the mixture column $h=0.2 \mathrm{~g}$ and $h=0.5 \mathrm{~m}$ (Fig. 5.4, a, curve 1 and curve 2), the amplitude 
of the oscillations decreases. In the resonance zone $h=0.3 \mathrm{~m}$ (Fig. 5.4, curve 3), the amplitude of oscillations increases, which is confirmed by the graph (Fig. 5.4, $\boldsymbol{b}$ ). A noticeable effect on the amplitude has the speed of propagation of oscillations (Fig. 5.4), the value of which is included in the determination of the numerical values of the wave coefficients $a$ and $d$ (5.7). So, an error in determining the vibration speed by $10 \%$ can lead to a discrepancy between the calculated and actual vibration amplitudes by $150 \%$ (Fig. 5.4, curve 2).

The degree of influence of active resistance forces (Fig. 5.4, $\boldsymbol{d}$ ) is determined by the height of the mixture column. In the zone of the greatest value of the vibration amplitude for a height of $h=0.3 \mathrm{~m}$ (curve 1, Fig. 5.4, $\boldsymbol{d}$ ), an increase $\gamma$ leads to a natural decrease $x_{0}$. For the height (curve 1 , Fig. 5.4, $\boldsymbol{d}$ ), the picture is the opposite: here an increase $\gamma$ leads to an increase in the amplitude of oscillations $x_{0}$.

The change $\gamma$ from 0.1 to 0.3 led to an increase in the $x_{0}$ amplitude from 0.156 to $0.173 \mathrm{~mm}$.

The considered examples clearly confirm the taken into account the reactive and active resistance of the concrete mixture.

\subsection{STUDY OF THE PARAMETERS OF SOIL COMPACTION PROCESSES UNDER THE MAIN PIPELINE}

Main pipelines are rightly considered the energy arteries of Ukraine, because they provide energy resources not only for the needs of the state's own economy, but also for the transportation of oil and gas to more than 15 European countries. The current state of ensuring reliable and safe operation of trunk pipelines requires the urgent development and implementation of new equipment and technologies for capital repairs of pipelines, the use of which will significantly accelerate the pace of repair work with a decrease in material and labor costs for their implementation [7-9].

A feature of the new technology is that after a complex of works on replacing insulation under the repaired pipeline, soil is poured and compacted under the pipe to such an extent that it excludes subsidence of the latter after repair.

A schematic diagram of soil compaction under the pipeline by two surfaces symmetrically located relative to the longitudinal axis of the pipeline, synchronously moving against each other along the required trajectory, is shown in Fig. 5.5. In the process of compaction, there is a mutual influence of stress diagrams in the soil from both soil-compaction surfaces, which leads to the formation of a generalized stress diagram in the zone of the vertical soil mass under the main pipeline.

The stress state of the massif depends on many factors, primarily on the magnitude of the applied external loads, load modes, physical and mechanical characteristics of the compacting soil, the size of the soil mass under the pipe, the shape and size of the trench, and the design parameters of the soil compacting working equipment.

The values of technological factors in this study are taken to be fixed. The diameter of the pipeline is taken $D_{p}=1220 \mathrm{~mm}$, as the most common in Ukraine. The weight of one running meter of the pipeline is assumed unchanged and maximum in value, for the case when the pipe is filled with 
oil $q_{p}=14.3 \mathrm{kN} / \mathrm{m}$. The height of the soil mass under the pipeline (the height of the placement of the lower point of the pipe relative to the bottom of the trench) is taken no more than $H=820 \mathrm{~mm}$. The geometrical dimensions of the trench for pipeline repair $D_{p}=1220 \mathrm{~mm}$, the following: depth $h=3000 \mathrm{~mm}$, width of the trench at the top $b_{t}=4400 \mathrm{~mm}$ and at the bottom $b_{b}=2620 \mathrm{~mm}$ (Fig. 5.5).

The speed of application of loads to the soil-packing surfaces $V_{1}$ reflects the mode of operation of the soil-packing equipment. It must be consistent with the overlap factor of the compaction zone $[10,11]$. Soil moisture $W$ has a significant effect on the characteristics of its compaction. Let's believe that the compacted soil has an optimal moisture content $W_{0}[12,13]$.

The structural and force factors that significantly affect the soil compaction process under the pipelines include the geometric dimensions of the sealing surfaces, their relative position in the trench relative to the pipeline and the pressure they create on the compacted soil mass. Flat undeformed surfaces (blades) of rectangular shape are taken as working surfaces.

Determining the approach to the study of the stress-strain state of the soil mass under the pipeline, let's note that in the elastic region the stresses are directly proportional to the value of the applied loads. With a known value of stresses in the soil mass from the action of each fixed pressure of the sealing blades on the soil, it is possible to determine the value of stresses for another value of the applied pressure to the soil mass $[14,15]$. Thus, with a known stress in the soil mass under the action of a fixed pressure $P$ created by the working surfaces, it is possible to find new stresses in the soil mass for other values of the applied pressure of the blades on the soil:

$$
\sigma^{\prime}=\frac{P^{\prime}}{P} \cdot \sigma,
$$

where $P$ - fixed pressure created by the blades on the soil mass; $\sigma$ - known value of stresses in the soil mass from the action of a fixed pressure.

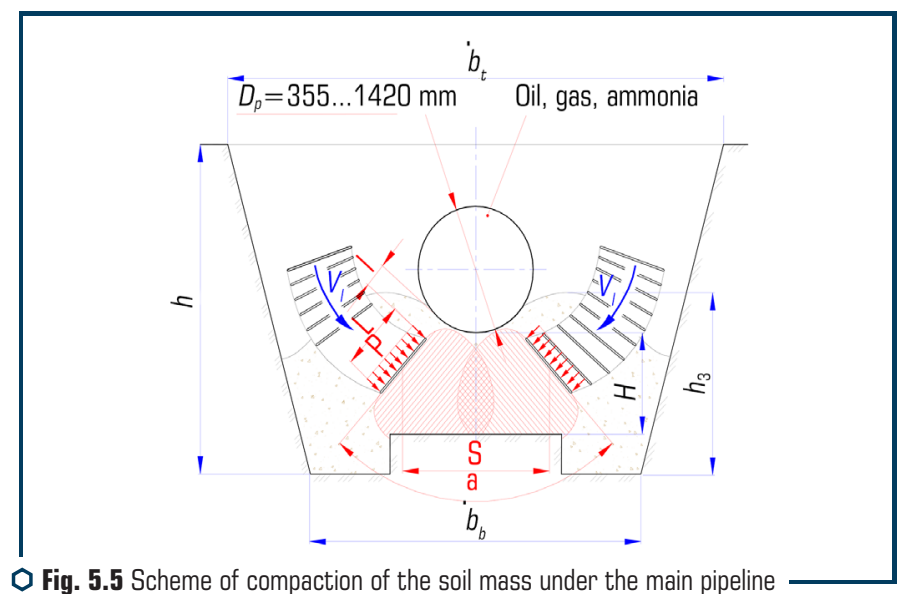


It follows from this that the pressure $P$ created by the blades on the soil can be excluded from the number of variable factors.

Since the pressure on the soil from the blade is distributed evenly both along the length and along its width, the problem of determining the stress-strain state of the soil under the pipe in the direction along the pipeline can be considered as flat. Consequently, the stresses in the soil, when they are determined within the framework of flat surrender, will depend on the value of the pressure under the blades, regardless of the width of the blades $B$. On the other hand, under all equal loading conditions (fixed pressures), when the width of the blade changes, the stresses in the soil mass will change proportional to the width of the blade $B$ :

$$
\sigma^{\prime}=\frac{B^{\prime}}{B} \cdot \sigma,
$$

where $\sigma^{\prime}$ - stress at an arbitrary blade width; $\sigma$ - stress determined for a fixed blade width.

Therefore, such a geometric parameter as the width of the blade $B$ is also referred to as constant factors.

Combining formulas (5.11) and (5.12), let's obtain the dependence of the stress $\sigma^{\prime}$ for arbitrary $P^{\prime}$ and $B^{\prime}$ at a known stress $\sigma$ arising in a flat body with thickness $B$ and a given load $P$ :

$$
\sigma^{\prime}=\sigma \cdot \frac{P^{\prime} B}{P B^{\prime}} .
$$

Thus, on the basis of consideration and analysis of the factors affecting the stress-strain state of the soil under the pipeline, it was established that the unchanged (constant) ones should include: $h=3000 \mathrm{~mm}, b_{t}=4400 \mathrm{~mm}, b_{b}=2620 \mathrm{~mm}, H=820 \mathrm{~mm}, D_{p}=1220 \mathrm{~mm}, q_{p}=14.3 \mathrm{kN} / \mathrm{m}$, $h_{d}=H+0.5 D_{p}, P=10, \mu=0.37$.

Consequently, the constant factors, the value of which must be determined experimentally, include: the compression rate $V_{1}$, the compaction delay time $t_{d}$, the compaction overlap coefficient $K_{0}$, the elastic modulus $E$, the optimal moisture content Wo.

In the process of studying the stress-strain state of the soil under the pipeline, it is necessary to determine the effect on the characteristics of the stress state of the following parameters: blade angle $\alpha$, blade length $L$ and width $B$, blade pressure $P$ on the ground, distance / from blade to pipe, as well as distance $S$ between blades (Fig. 5.6). Determination of the rational values of these factors and their interaction in the process of soil compaction is the main task of the study.

The essence of the study is to determine the effect of changes in the linear parameters $L$ and $B$ of the sealing blades, the pressure $P$ they create on the ground, on the provision of the necessary stresses in the soil mass under the pipeline, taking into account the parameters $\alpha, I$ and $S$, characterizing the position of the blades in the trench relative to the pipeline of a given diameter.

To construct a mathematical model of the stress state of the soil mass under the pipeline, let's select the values of the variable factors characterizing the compaction process for the zero level and the limits of change of these values are the intervals of variation of the factors. The value 
of the variation intervals is chosen based on the following considerations and requirements. The compression angle was chosen $\alpha=90^{\circ} \ldots 70^{\circ}$, based on the values of the angle of the prism of the soil massif under the pipeline, at which a sufficient stability coefficient of the massif will be ensured. When choosing the intervals for changing the linear parameters of the blades $L$ and $B$, we leave, first of all, from the position of ensuring guaranteed non-damage of the pipeline insulation coating during soil compaction $L=450 \ldots 650 \mathrm{~mm}$.

Based on the results of studies of soil compaction with stamps, the recommended width of the stamp should be equal to half of its length, because it is with this ratio that the maximum depth of the active compaction zone, $B=225 \ldots 325 \mathrm{~mm}$, is achieved during the compaction process. In further studies, let's take the value of the blade width to be constant and equal to $B=300 \mathrm{~mm}$.

Since it is known that the depth of the core during soil compaction [13] is equal to two dimensions of the smaller side of the stamp [11], and taking into account the predicted increase in the active zones as a result of their overlap as a result of the oncoming motion of the sealing blades, let's take $S=1200 \ldots 1600 \mathrm{~mm}$. The levels of the selected factors and the intervals of their variation are presented in Table $\mathbf{5 . 2}$.

Table 5.2 Factor levels and intervals of their variation study of the stress state of the MP soil bed

\begin{tabular}{llllll}
\hline \multirow{2}{*}{ Independent variables } & Variation levels & \multicolumn{2}{l}{$\begin{array}{l}\text { Variation } \\
\text { intervals } \Delta \boldsymbol{i}\end{array}$} \\
\cline { 3 - 6 } & Upper $+\mathbf{1}$ & Basic $\mathbf{0}$ & Low $\mathbf{- 1}$ & 10 \\
\hline$X 1$ & $\alpha$, degree & 90 & 80 & 70 & 200 \\
$X 2$ & S, mm & 1600 & 1400 & 1200 & 100 \\
$X 3$ & L, mm & 650 & 550 & 450 & 50 \\
$X 4$ & I, mm & 250 & 200 & 150 & \\
\hline
\end{tabular}

The design diagram of soil compaction under the pipeline is shown in Fig. 5.6. The geometrical dimensions of the investigated soil massif were determined on the basis of the dimensions $b_{t}, b_{b}$, $h$ of the trench profile, built by the pipeline opening machine $D_{p}=1220 \mathrm{~mm}$. The height of the soil massif under the pipe $H$ is taken in accordance with the size of the developed soil massif under the pipe $H=820 \mathrm{~mm}$.

The soil mass, compacted under the pipeline, is bounded by two inclined surfaces symmetrical about the vertical axis passing through the center of the pipeline, the positions of which are regulated by the values of the compression angle $X 1$ and the distance between the blades $X 2$ (Fig. 5.7). It is accepted that the value of the parameter $X 2$ is calculated horizontally, located relative to the lower point of the pipeline at a distance of $1 / 3 \mathrm{H}$.

In this case, the mass of compacted soil is limited from the top of the generatrix of the pipe of a given diameter $D_{p}$, and from the bottom by the bottom of the excavated recess, which is located at a distance $H$ from the lower point of the generatrix of the pipeline. Since the pipeline wall and trench walls have significantly higher elastic moduli than the compacted soil mass, they can be 
considered undeformed surfaces. Therefore, in the zones where the compacted soil borders on the generatrix of the pipeline and the walls of the trench, it is legitimate to impose boundary conditions for the movement of the soil perpendicular to the undeformed surfaces.

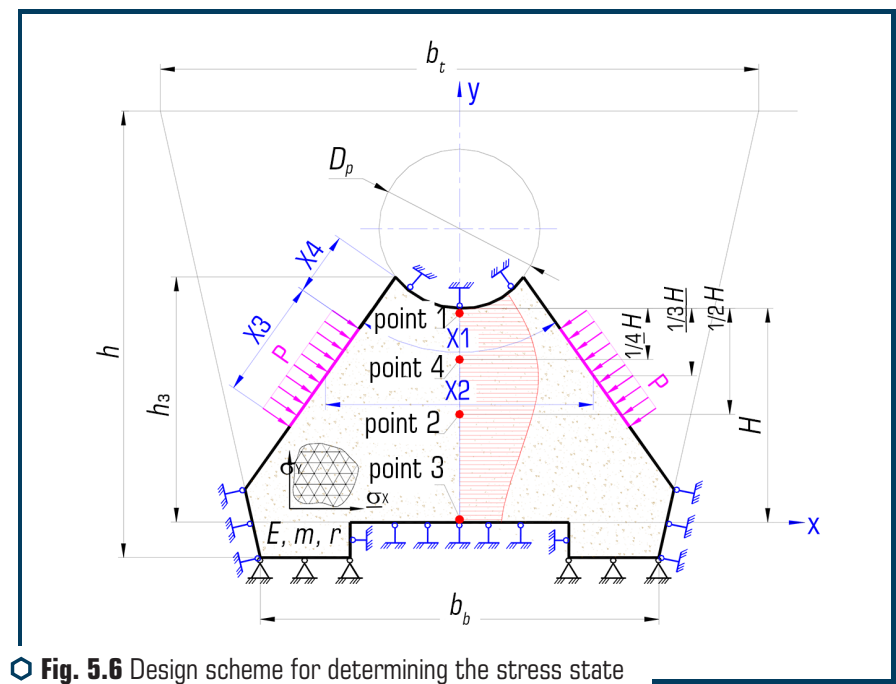
of the soil mass under the pipeline

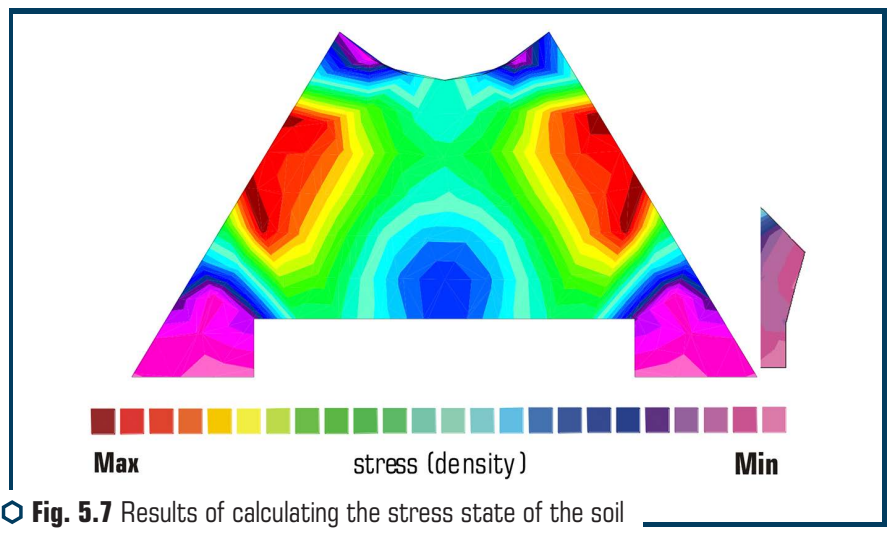
mass under the pipeline for typical experimental plans

Taking into account the above, for further research let's take soil compacted isotropic in volume.

The sizes, which are determined (reviews) in the study, are equivalent stresses at the characteristic points of the soil mass along the axis under the pipeline. Typical points are considered (Fig. 5.7): 
$Y 1$ - the magnitude of stresses in the soil mass in the area under the pipeline; $Y 2$ - the value of stresses in the middle part of the soil massif at a distance of 1/2N from the pipeline; $Y 3$ - the magnitude of stresses in the lower part of the massif at the level with the bottom of the trench; $Y 4$ - the value of stress in the soil at a distance of $1 / 4 \mathrm{H}$ from the pipeline.

Determination of the rational values of the parameters $X 1 \ldots X 4, B$ and $P$, their functional interdependence and influence on the stress state of the soil compacted under the pipeline made it possible to provide the necessary values of the soil compaction coefficients and the uniformity of its compaction.

The visual presentation of the results of calculating the stress state of the soil [8] under the pipeline using the finite element method made it possible to evaluate the influence of the interaction of compaction nuclei in the soil mass created by the blades of the working body, under the action of various combinations of values of the initial parameters that determine the compaction process. Typical results of calculating the stress state of the soil indicate that there may be combinations of initial parameters at which the expected effect of superposition of compaction nuclei is not observed, the stresses in the soil mass at different depths under the pipeline are insignificant, therefore, the coefficients of soil compaction remain minimal. The combination of the initial parameters (Fig. 5.8) provides a more uniform soil compaction under the pipeline, which indicates the presence of the effect of the interaction of compaction nuclei in the soil.

Thus, it can be argued that by changing the combinations of the initial parameters that determine the process of soil compaction under the pipeline, it is possible to control the interaction of soil compaction nuclei, as well as to influence the value of the final indicators of this process [11, 12, 16-18].

As a result of processing the data of numerical experiments [10], in accordance with the folded matrix, mathematical models were obtained to determine the stress state of the soil at the characteristic points of the massif under the pipelines, which are predictive models. Their adequacy was confirmed by the Fisher test with a confidence level of 0.95 [11].

$$
\begin{aligned}
& \sigma_{1}=\frac{P^{\prime} B}{P B^{\prime}} \cdot\left(\begin{array}{l}
0.08637-0.01696 \cdot x_{1}+0.02101 \cdot x_{2}- \\
-0.02524 \cdot x_{3}-0.00306 \cdot x_{1}^{2}+0.00367 \cdot x_{4}^{2}
\end{array}\right) ; \\
& \sigma_{2}=\frac{P^{\prime} B}{P B^{\prime}} \cdot\left(0.09741-0.0027 \cdot x_{1}-0.00798 \cdot x_{2}+0.01312 \cdot x_{3}+0.0024 \cdot x_{2}^{2}\right) ; \\
& \sigma_{3}=\frac{P^{\prime} B}{P B^{\prime}} \cdot\left(0.0725-0.00509 \cdot x_{2}-0.01162 \cdot x_{3}-0.00276 \cdot x_{1}^{2}\right) ; \\
& \sigma_{4}=\frac{P^{\prime} B}{P B^{\prime}} \cdot\left(0.09889-0.00642 \cdot x_{1}+0.00605 \cdot x_{3}-0.01496 \cdot x_{4}+0.00306 \cdot x_{2}^{2}\right) .
\end{aligned}
$$

Thanks to the predictive models (5.14)-(5.17), it became possible to control the process of interaction of the compaction nuclei by changing the linear dimensions of the blades, the parameters 
of their installation, namely: the compression angle $\alpha=X 1$, the distance between the blades $S=X 2$, the blade length $L=X 3 \mathrm{~mm}$, the distance from the blade to the pipeline $I=X 4$, blade width $B$.

Based on the solution of the system of equations (5.14)-(5.17), a rational combination of the output parameters of the soil compaction process under the pipelines was found: $\alpha=75^{\circ}$, $S=1400 \mathrm{~mm}, L=600 \mathrm{~mm}, I=180 \mathrm{~mm}, B=300 \mathrm{~mm}$, as well as the dependence for the characteristic points $1 \ldots 4$ of the change in the values of the compaction coefficients on the pressure of the blades on the ground is built (Fig. 5.8).

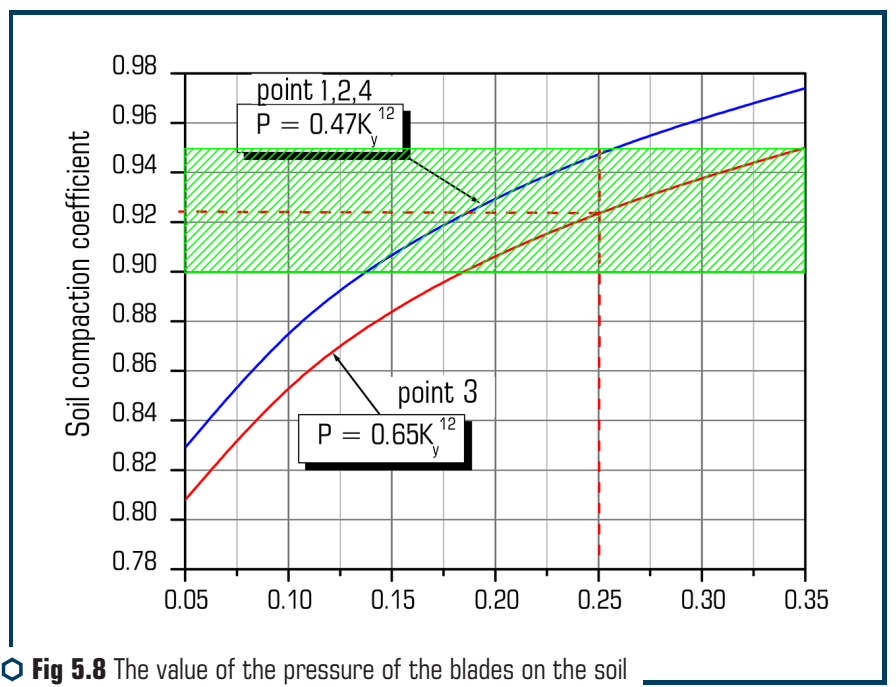
from the coefficient of its compaction

This made it possible to obtain the value of the pressure of the blades on the soil $P=0.2 \ldots . .0 .35 \mathrm{MPa}$, at which the necessary values of the soil compaction coefficients under the pipeline will be provided within the range of $K_{y}=0.9 \ldots 0.95$.

Rational values of the parameters of the working body and operating modes under which the interaction of soil compaction nuclei under the pipeline is guaranteed, adopted for subsequent research and for use in the design of working equipment for soil compaction under the pipeline.

\subsection{DISCUSSION OF RESEARCH RESULTS}

A certain model of the processing medium, based on a general view of the compaction process, is characterized by three stages. This is at the first stage of compaction of a granular medium (Fig. 5.1), at the second and third stages of compaction, an elastic-viscoplastic medium. 
This important result requires a clear definition of the physical and mathematical model, which is suggested by equations (5.1). Further studies are required to determine the temporal assessment of the course of these stages. Application of the idea of considering the "technical system - medium" system as a system that at any moment of movement consists of two components. One is capable of storing energy that passes from one form to another (reactance), and the other is capable of dissipating energy (resistance). A certain significant influence of the components of resistance on the movement of a technical system (Fig. 5.4) testifies to the hypothesis put forward about the need to take into account the forces of resistance of the medium. A technical system is a system with discrete parameters, and a medium with distributed parameters. In this case, this general system is reduced to a computational system in the form of a system with discrete parameters, in which the wave phenomena of the medium are preserved and are represented by a contact force. The obtained wave coefficients (5.7) have general properties. They can be used in any equations of motion for such technical systems. This is the novelty of the method of analytical study of vibration systems for compaction of mixtures.

The presentation of the results of calculating the stress state of the soil under the pipeline using the finite element method made it possible to assess the influence of the interaction of compaction of nuclei in the soil mass. By changing the combinations of the initial parameters that determine the process of soil compaction under the pipeline, it allows to control the interaction of soil compaction nuclei, as well as to influence the value of the final indicators of this process. Based on the results of studies of soil compaction with stamps, the width of the stamp was determined $(B=225 \ldots 325 \mathrm{~mm}$ ). Its value is equal to half of its length, since according to this ratio, the maximum depth of the active zone of compaction is achieved during the compaction process. A rational combination of the initial parameters of the soil compaction process under the pipelines $\left(\alpha=75^{\circ}, S=1400 \mathrm{~mm}, L=600 \mathrm{~mm}, I=180 \mathrm{~mm}, B=300 \mathrm{~mm}\right.$ ) has been established under which the interaction of soil compaction nuclei under the pipeline is guaranteed.

\section{CONCLUSIONS TO SECTION 5}

1. The model of the processing medium, characterized by three stages of compaction, has been determined.

2. The review and analysis of physical and mathematical models of the process of compaction of technological media has been carried out.

3. A model of the «vibration plant - medium» hybrid system is proposed, in which active and reactive resistances to wave coefficients are taken into account.

4. The results of calculations of changes in the parameters of vibration machines for the processes of compaction of concrete mixture have been obtained, confirming the implementation of accounting for the reactive and active resistance of the concrete mixture. 
5. Predictive mathematical models have been created to determine the stress state of the soil during compaction at the characteristic points of the massif under the pipelines, which make it possible to obtain rational values of the parameters of the working bodies for soil compaction.

6. Regularities of movement of technical systems for compacting concrete mixtures and soils and their rational parameters have been established.

7. An algorithm and methodology for calculating the main parameters of vibration systems for compaction of working media have been developed.

\section{REFERENCES}

1. Nazarenko, I., Gaidaichuk, V., Dedov, O., Diachenko, 0. (2017). Investigation of vibration machine movement with a multimode oscillation spectrum. Eastern-European Journal of Enterprise Technologies, 6 (1 (90)), 28-36. doi: http://doi.org/10.15587/1729-4061.2017.118731

2. Nazarenko, I., Gaidaichuk, V., Dedov, O., Diachenko, 0. (2018). Determination of stresses and strains in the shaping structure under spatial load. Eastern-European Journal of Enterprise Technologies, 6 (7 (96)), 13-18. doi: http://doi.org/10.15587/1729-4061.2018.147195

3. Nazarenko, I., Ruchynskyi, M., Delembovskyi, M. (2018). The Basic Parameters of Vibration Settings for Sealing Horizontal Surfaces. International Journal of Engineering \& Technology, 7 (3.2), 255-259. doi: http://doi.org/10.14419/ijet.v7i3.2.14415

4. Nesterenko, M., Nazarenko, I., Molchanov, P. (2018). Cassette Installation with Active Working Body in the Separating Partition. International Journal of Engineering \& Technology, 7 (3.2), 265-268. doi: http://doi.org/10.14419/ijet.v7i3.2.14417

5. Nazarenko, I. I., Ruchynskyi, M. M., Sviderskyi, A. T., Kobylanska, I. M., Harasim, D., Kalizhanova, A., Kozbakova, A. (2019). Development of energy-efficient vibration machines for the buiding-and-contruction industry. Przeglad Elektrotechniczny, 95 (4), 53-59. doi: http:// doi.org/10.15199/48.2019.04.10

6. Klets, D., Gritsuk, I. V., Makovetskyi, A., Bulgakov, N., Podrigalo, M., Kyrychenko, I. et. al. (2018). Information Security Risk Management of Vehicles. SAE Technical Paper Series. doi: http:// doi.org/10.4271/2018-01-0015

7. Dubovenko, Y. I., Kuzminets, M. P. (2017). The experience of integrating of GIS techniques in the construction of digital maps of geophysical fields. 16th International Conference on Geoinformatics - Theoretical and Applied Aspects. doi: http://doi.org/10.3997/2214-4609.201701851

8. Dubovenko, Y. I., Shumlianska, L. A., Kuzminets, M. P. (2020). Seismic velocity gradient stratification of the mantle at Ukrainian Shield. Geoinformatics: Theoretical and Applied Aspects 2020. doi: http://doi.org/10.3997/2214-4609.2020geo063

9. Dubovenko, Y. I., Chorna, O. A., Kuzminets, M. P. (2020). Modeling of the potential fields transformants for the ring structure Illinetska. Geoinformatics: Theoretical and Applied Aspects 2020. doi: http://doi.org/10.3997/2214-4609.2020geo062 
10. Nazirova, A. B., Dubovenko, Y. I., Abdoldina, F. N., Kuzminets, M. P. (2021). Optimization of GIS modules for processing data of gravity monitoring of subsoil in the Republic of Kazakhstan. Geoinformatics. doi: http://doi.org/10.3997/2214-4609.20215521136

11. Nazarenko, I., Svidersky, A., Kostenyuk, A., Dedov, O., Kyzminec, N., Slipetskyi, V. (2020). Determination of the workflow of energy-saving vibration unit with polyphase spectrum of vibrations. Eastern-European Journal of Enterprise Technologies, 1 (7 (103)), 43-49. doi: http://doi.org/10.15587/1729-4061.0.184632

12. Onishchenko, A., Koretskyi, A., Bashkevych, I., Ostroverkh, B., Bieliatynskyi, A. (2020). Dam Failure Model and Its Influence on the Bridge Construction. Advances in Intelligent Systems and Computing, 229-237. doi: http://doi.org/10.1007/978-3-030-57450-5_21

13. Onishchenko, A., Lapchenko, A., Fedorenko, O., Bieliatynskyi, A. (2020). Research of the Properties of Bitumen Modified by Polymer Latex. Advances in Intelligent Systems and Computing, 104-116. doi: http://doi.org/10.1007/978-3-030-57450-5_10

14. Kovalchuk, V., Kravets, I., Nabochenko, O., Onyshchenko, A., Fedorenko, O., Pentsak, A. et. al. (2021). Devising a procedure for assessing the subgrade compaction degree based on the propagation rate of elastic waves. Eastern-European Journal of Enterprise Technologies, 1 (5 (109)), 6-15. doi: http://doi.org/10.15587/1729-4061.2021.225520

15. Luchko, J., Kovalchuk, V., Kravets, I., Gajda, O., Onyshchenko, A. (2020). Determining patterns in the stresseddeformed state of the railroad track subgrade reinforced with tubular drains. Eastern-European Journal of Enterprise Technologies, 5 (7 (107)), 6-13. doi: http:// doi.org/10.15587/1729-4061.2020.213525

16. Lantukh-Lyashchenko, A., Onishchenko, A., Davydenko, 0. (2020). Problem of the degradation criteria for transportation construction elements. E3S Web of Conferences, 164 , 03014. doi: http://doi.org/10.1051/e3sconf/202016403014

17. Nazarenko, I., Dedov, O., Bernyk, I., Rogovskii, I., Bondarenko, A., Zapryvoda, A. et. al. (2020). Determining the regions of stability in the motion regimes and parameters of vibratory machines for different technological purposes. Eastern-European Journal of Enterprise Technologies, 6 (7 (108)), 71-79. doi: http://doi.org/10.15587/1729-4061.2020.217747

18. Nazarenko, I., Gavryukov, O., Klyon, A., Ruchynsky, N. (2018). Determination of the optimal parameters of a tubular belt conveyor depending on such an economical. Eastern-European Journal of Enterprise Technologies, 3 (1 (93)), 34-42. doi: http://doi.org/10.15587/17294061.2018.131552 